\title{
ON NEW CHEBYSHEV INEQUALITIES VIA FRACTIONAL OPERATORS
}

\author{
EMRULLAH AYKAN ALAN, BARIŞ ÇELIK, ERHAN SET, AND ZOUBIR DAHMANI
}

Received 27 December, 2020

\begin{abstract}
The aim of this paper is to establish several fractional integral inequalities related to the weighted and the extended Chebyshev functional. We use generalized fractional integral operators, new conformable fractional integral operators and Saigo fractional integral operators to prove our results. This study states that our findings are more convenient and efficient than other available results.
\end{abstract}

2010 Mathematics Subject Classification: 26A33; 26D10; 26D15

Keywords: Chebyshev inequality, generalized fractional integral operators, new conformable fractional integral operators, Saigo fractional integral operators

\section{INTRODUCTION AND PRELIMINARIES}

The integral inequality theory is very important in applied sciences. For more details, we refer the reader to [1,2] and the references therein. Moreover, the study of the integral inequalities using fractional integration theory is also of great importance, we refer to $[8,9,14,15]$ for some applications. In this paper, we will be concerned with the extended and the weighted Chebyshev functional [2,9].

So, in this section, we introduce some definitions and some published results that have motivated the present work; we begin by recalling the following definition.

Definition 1. If $f$ and $g$ are two integrable functions on $[a, b]$ and $p$ is a positive and integrable function on $[a, b]$, Chebyshev functional is given by the following quantity

$$
\mathrm{T}(f, g, p):=\int_{a}^{b} p(x) d x \int_{a}^{b} f(x) g(x) p(x) d x-\int_{a}^{b} f(x) p(x) d x \int_{a}^{b} g(x) p(x) d x .
$$

Related to the above quantity, in [6], N. Elezovic et al. proved the following integral result: 
Theorem 1. [6, Theorem B.] The inequality

$$
\begin{aligned}
|\mathrm{T}(f, g, p)| \leq & \frac{1}{2}\left(\left.\left.\int_{a}^{b} \int_{a}^{b} p(x) p(y)|x-y|^{\frac{1}{\alpha^{\prime}}+\frac{1}{\beta^{\prime}}}\left|\int_{y}^{x}\right| f^{\prime}(t)\right|^{\alpha} d t\right|^{\frac{\gamma}{\alpha}} d x d y\right)^{\frac{1}{\gamma}} \\
& \times\left(\left.\left.\int_{a}^{b} \int_{a}^{b} p(x) p(y)|x-y|^{\frac{1}{\alpha^{\prime}}+\frac{1}{\beta^{\prime}}}\left|\int_{y}^{x}\right| g^{\prime}(t)\right|^{\beta} d t\right|^{\frac{\gamma}{\beta}} d x d y\right)^{\frac{1}{\gamma}} \\
\leq & \frac{1}{2}|| f^{\prime}\left\|_{\alpha}\right\|_{g^{\prime}} \|_{\beta}\left(\int_{a}^{b} \int_{a}^{b} p(x) p(y)|x-y|^{\frac{1}{\alpha^{\prime}}+\frac{1}{\beta^{\prime}}} d x d y\right)
\end{aligned}
$$

is valid provided that $f^{\prime} \in \mathrm{L}^{\alpha}([a, b])$ and $g^{\prime} \in \mathrm{L}^{\beta}([a, b]) ; \alpha>1, \beta>1, \gamma>1$, $\alpha^{-1}+\alpha^{\prime^{-1}}=1, \beta^{-1}+\beta^{\prime^{-1}}=1, \gamma^{-1}+\gamma^{\prime^{-1}}=1$.

Then, by means of the following fractional integral definition:

Definition 2. Let $f \in L_{1}[a, b]$. The Riemannn-Liouville integral of order $a \geq 0$ is defined by

$$
J_{a}^{\alpha} f(x)=\frac{1}{\Gamma(\alpha)} \int_{a}^{x}(x-t)^{\alpha-1} f(t) d t, \quad a \leq x \leq b .
$$

The authors of the paper [5] established the following theorem:

Theorem 2. [5, Theorem 3.1]

$$
\begin{aligned}
2 \mid \mathbf{J}^{\sigma} p(t) \mathbf{J}^{\sigma} p f g(t) & -\mathbf{J}^{\sigma} p f(t) \mathbf{J}^{\sigma} p g(t) \mid \\
& \leq \frac{\left\|f ^ { \prime } \left|\left\|_{\alpha}|| g^{\prime}\right\|_{\beta}\right.\right.}{\Gamma^{2}(\sigma)} \int_{0}^{t} \int_{0}^{t}(t-x)^{\sigma-1}(t-y)^{\sigma-1}|x-y| p(x) p(y) d x d y,
\end{aligned}
$$

where $f^{\prime} \in \mathrm{L}^{\alpha}([0, \infty])$ and $g^{\prime} \in \mathrm{L}^{\beta}([0, \infty]) ; \alpha>1, \beta>1, \alpha^{-1}+\beta^{-1}=1$.

Furthermore, by considering a second weighted function $q$ (which is supposed to be positive and integrable) on $[a, b]$, we consider the extended Chebyshev's functional $[3,9]$ :

$$
\begin{aligned}
\tilde{\mathrm{T}}(f, g, p, q):= & \int_{a}^{b} q(x) d x \int_{a}^{b} p(x) f(x) g(x) d x+\int_{a}^{b} p(x) d x \int_{a}^{b} q(x) f(x) g(x) d x \\
& -\int_{a}^{b} p(x) f(x) d x \int_{a}^{b} q(x) g(x) d x-\int_{a}^{b} q(x) f(x) d x \int_{a}^{b} p(x) g(x) d x .
\end{aligned}
$$

Then, we recall the following two theorems [4].

Theorem 3. [4, Theorem 3.1] Let $f$ and $g$ be two differentiable functions on $[0, \infty)$ and $p$ be positive and integrable function on $[0, \infty)$. If $f^{\prime} \in L^{\alpha}([0, \infty)), g^{\prime} \in L^{\beta}([0, \infty))$, 
$\alpha, \beta, \gamma>1$ with $\frac{1}{\alpha}+\frac{1}{\alpha^{\prime}}=1, \frac{1}{\beta}+\frac{1}{\beta^{\prime}}=1$ and $\frac{1}{\gamma}+\frac{1}{\gamma^{\prime}}=1$, then for all $t>0, \delta>0$, we have the inequality

$$
\begin{aligned}
& 2\left|J^{\delta} p(t) J^{\delta} p f g(t)-J^{\delta} p g(t) J^{\delta} p f(t)\right| \\
& \leq\left(\frac{\left\|f^{\prime} \mid\right\|_{\alpha}^{\gamma}}{\Gamma(\delta)} \int_{0}^{t} \int_{0}^{t}(t-x)^{\delta-1}(t-y)^{\delta-1} p(x) p(y)|x-y|^{\frac{1}{\alpha^{\prime}}+\frac{1}{\beta^{\prime}}} d x d y\right)^{\frac{1}{\gamma}} \\
& \quad \times\left(\frac{\left\|g^{\prime} \mid\right\|_{\beta}^{\gamma}}{\Gamma(\alpha)} \int_{0}^{t} \int_{0}^{t}(t-x)^{\delta-1}(t-y)^{\delta-1} p(x) p(y)|x-y|^{\frac{1}{\alpha^{\prime}}+\frac{1}{\beta^{\prime}}} d x d y\right)^{\frac{1}{\gamma^{\prime}}} \\
& \leq \frac{\left\|f^{\prime}\right\|_{\alpha}\left\|g^{\prime}\right\|_{\beta}}{\Gamma(\delta)^{2}}\left(\int_{0}^{t} \int_{0}^{t}(t-x)^{\delta-1}(t-y)^{\delta-1}|x-y|^{\frac{1}{\alpha^{\prime}}+\frac{1}{\beta^{\prime}}} p(x) p(y) d x d y\right) .
\end{aligned}
$$

Theorem 4. [4, Theorem 3.3] Let $f$ and $g$ be two differentiable functions on $[0, \infty)$ and $p, q$ be two positive and integrable function on $[0, \infty)$. If $f^{\prime} \in L^{\alpha}([0, \infty))$, $g^{\prime} \in L^{\beta}([0, \infty)), \alpha, \beta, \gamma>1$ with $\frac{1}{\alpha}+\frac{1}{\alpha^{\prime}}=1, \frac{1}{\beta}+\frac{1}{\beta^{\prime}}=1$ and $\frac{1}{\gamma}+\frac{1}{\gamma^{\prime}}=1$, then for all $t>0, \delta>0$, we have:

$$
\begin{aligned}
& \left|J^{\delta} q(t) J^{\delta} p f g(t)+J^{\delta} p(t) J^{\delta} q f g(t)-J^{\delta} p f(t) J^{\delta} q g(t)-J^{\delta} q f(t) J^{\delta} p g(t)\right| \\
& \leq \frac{\left\|f^{\prime}\right\|_{\alpha}\left\|g^{\prime}\right\|_{\beta}}{\Gamma(\delta)^{2}}\left(\int_{0}^{t} \int_{0}^{t}(t-x)^{\delta-1}(t-y)^{\delta-1}|x-y|^{\frac{1}{\alpha^{\prime}}+\frac{1}{\beta^{\prime}}} p(x) q(y) d x d y\right) .
\end{aligned}
$$

The main aim of this paper is to establish new fractional integral inequalities related to the weighted and the extended Chebyshev functional. We use the generalized fractional integral operator to establish some of our main results. Then, by considering the conformable fractional integral approach, we establish other integral results. Finally, by Saigo fractional integral operator, we present to the reader other results on integral inequalities. Some published results related to integral inequalities in the sense or Riemann-Liouville $[4,5]$ are deduced as some special cases.

\section{MAIN RESUltS}

Let's first recall the generalized fractional integral operators definition we used in the proof of Theorem 5 and Theorem 6.

In [10], Raina introduced a generalized class of Mittag-Leffler functions defined formally by

$$
\mathcal{F}_{\rho, \lambda}^{\sigma}(x)=\mathcal{F}_{\rho, \lambda}^{\sigma(0), \sigma(1), \ldots}(x)=\sum_{k=0}^{\infty} \frac{\sigma(k)}{\Gamma(\rho k+\lambda)} x^{k} \quad(\rho, \lambda>0 ;|x|<\mathbf{R}),
$$

where the coefficients $\sigma(k),(k \in \mathbb{N}=\mathbb{N} \cup\{0\})$ is a bounded sequence of positive real numbers and $\mathbf{R}$ is the set of real numbers. With the help of (2.1), Raina [10] and 
Agarwal et al. [1] defined the following left-sided and right-sided fractional integral operators respectively, as follows:

$$
\begin{array}{ll}
\left(g_{\rho, \lambda, a+; w}^{\sigma} \varphi\right)(x)=\int_{a}^{x}(x-t)^{\lambda-1} \mathcal{F}_{\rho, \lambda}^{\sigma}\left[w(x-t)^{\rho}\right] \varphi(t) d t & (x>a), \\
\left(g_{\rho, \lambda, b-; w}^{\sigma} \varphi\right)(x)=\int_{x}^{b}(t-x)^{\lambda-1} \mathcal{F}_{\rho, \lambda}^{\sigma}\left[w(t-x)^{\rho}\right] \varphi(t) d t & (x<b),
\end{array}
$$

where $\lambda, \rho>0, w \in \mathbb{R}$ and $\varphi(t)$ is such that the integral on the right side exits. In recently some integral inequalities this operator involving have appeared in the literature (see, e.g., [13-17]).

It is easy to verify that $g_{\rho, \lambda, a+; w}^{\sigma} \varphi(t)$ and $g_{\rho, \lambda, b-; w}^{\sigma} \varphi(t)$ are bounded integral operators on $L(a, b)$, if

$$
\mathfrak{M}:=\mathcal{F}_{\rho, \lambda+1}^{\sigma}\left[w(b-a)^{\rho}\right]<\infty .
$$

In fact, for $\varphi \in L(a, b)$, we have

$$
\left\|\mathcal{J}_{\rho, \lambda, a+; w}^{\sigma} \varphi(x)\right\|_{1} \leq \mathfrak{M}(b-a)^{\lambda}\|\varphi\|_{1}
$$

and

$$
\left\|\mathcal{I}_{\rho, \lambda, b-; w}^{\sigma} \varphi(x)\right\|_{1} \leq \mathfrak{M}(b-a)^{\lambda}\|\varphi\|_{1}
$$

where

$$
\|\varphi\|_{p}:=\left(\int_{a}^{b}|\varphi(x)|^{p} d x\right)^{\frac{1}{p}} .
$$

Here, many useful fractional integral operators can be obtained by specializing the coefficient $\sigma(k)$. For instance the classical Riemann-Liouville fractional integrals $J_{a+}^{\alpha}$ and $J_{b-}^{\alpha}$ of order $\alpha$ follow easily by setting $\lambda=\alpha, \sigma(0)=1$ and $w=0$ in (2.2) and (2.3).

We begin our main results by proving the following theorem.

Theorem 5. Let $f$ and $g$ be two differentiable functions on $[0, \infty)$ and $p$ be positive and integrable function on $[0, \infty)$. If $f^{\prime} \in L_{r}[a, b], g^{\prime} \in L_{s}[a, b], r, s, \gamma>1$ with $\frac{1}{r}+\frac{1}{r^{\prime}}=1, \frac{1}{s}+\frac{1}{s^{\prime}}=1$ and $\frac{1}{\gamma}+\frac{1}{\gamma^{\prime}}=1$, then for all $t>0, \alpha, \rho, w \geq 0$, we have;

$$
\begin{aligned}
& 2\left|g_{\rho, \lambda, a+; w}^{\sigma} p(t) \mathcal{I}_{\rho, \lambda, a+; w}^{\sigma} p f g(t)-g_{\rho, \lambda, a+; w}^{\sigma} p g(t) g_{\rho, \lambda, a+; w}^{\sigma} p f(t)\right| \\
& \leq\left[\left\|f^{\prime}\right\|_{r}^{\gamma} \int_{a}^{t} \int_{a}^{t}(t-x)^{\lambda-1}(t-y)^{\lambda-1} \mathcal{F}_{\rho, \lambda}^{\sigma}\left[w(t-x)^{\rho}\right] \mathcal{F}_{\rho, \lambda}^{\sigma}\left[w(t-y)^{\rho}\right]\right. \\
& \left.\quad \times|x-y|^{\frac{1}{r^{\prime}}+\frac{1}{s^{\prime}}} p(x) p(y) d x d y\right]^{\frac{1}{\gamma}}
\end{aligned}
$$




$$
\begin{aligned}
& \times\left[\| g^{\prime}||_{s}^{\gamma} \int_{a}^{t} \int_{a}^{t}(t-x)^{\lambda-1}(t-y)^{\lambda-1} \mathcal{F}_{\rho, \lambda}^{\sigma}\left[w(t-x)^{\rho}\right] \mathcal{F}_{\rho, \lambda}^{\sigma}\left[w(t-y)^{\rho}\right]\right. \\
& \left.\quad \times|x-y|^{\frac{1}{r^{+}}+\frac{1}{s^{\prime}}} p(x) p(y) d x d y\right]^{\frac{1}{\gamma}} \\
& \leq|| f^{\prime}|| r|| g^{\prime} \|_{s} \int_{a}^{t} \int_{a}^{t}(t-x)^{\lambda-1}(t-y)^{\lambda-1} \mathcal{F}_{\rho, \lambda}^{\sigma}\left[w(t-x)^{\rho}\right] \mathcal{F}_{\rho, \lambda}^{\sigma}\left[w(t-y)^{\rho}\right] \\
& \quad \times|x-y|^{\frac{1}{r^{1}}+\frac{1}{s^{\prime}}} p(x) p(y) d x d y .
\end{aligned}
$$

Proof. We consider the function $H$ defined by:

$$
H(x, y):=(f(x)-f(y)(g(x)-g(y))) ; \quad x, y \in(0, t), t>0 .
$$

Some easy techniques allow us to write

$$
\begin{aligned}
& \int_{a}^{t}(t-x)^{\lambda-1} \mathcal{F}_{\rho, \lambda}^{\sigma}\left[w(t-x)^{\rho}\right] p(x) H(x, y) d x=g_{\rho, \lambda, a+; w}^{\sigma} p f g(t) \\
& -g(y) g_{\rho, \lambda, a+; w}^{\sigma} p f(t)-f(y) \mathcal{g}_{\rho, \lambda, a+; w}^{\sigma} p g(t)+f(y) g(y) g_{\rho, \lambda, a+; w}^{\sigma} p(t) .
\end{aligned}
$$

By the same arguments as above, it yields that

$$
\begin{array}{r}
\int_{a}^{t} \int_{a}^{t}(t-x)^{\lambda-1}(t-y)^{\lambda-1} \mathcal{F}_{\rho, \lambda}^{\sigma}\left[w(t-x)^{\rho}\right] \mathcal{F}_{\rho, \lambda}^{\sigma}\left[w(t-y)^{\rho}\right] p(x) p(y) H(x, y) d x d y \\
=2\left[\mathcal{g}_{\rho, \lambda, a+; w}^{\sigma} p(t) \mathcal{g}_{\rho, \lambda, a+; w}^{\sigma} p f g(t)-\mathcal{g}_{\rho, \lambda, a+; w}^{\sigma} p g(t) \mathcal{g}_{\rho, \lambda, a+; w}^{\sigma} p f(t)\right] .
\end{array}
$$

Also, we can see that

$$
H(x, y):=\int_{y}^{x} \int_{y}^{x} f^{\prime}(u) g^{\prime}(w) d u d w .
$$

By using Hölder inequality, we have

$$
|f(x)-f(y)| \leq\left.\left.|x-y|^{\frac{1}{r^{\prime}}}\left|\int_{y}^{x}\right| f^{\prime}(u)\right|^{r} d u\right|^{\frac{1}{r}}
$$

and

$$
|g(x)-g(y)| \leq\left.\left.|x-y|^{\frac{1}{s^{f}}}\left|\int_{y}^{x}\right| g^{\prime}(w)\right|^{s} d w\right|^{\frac{1}{s}} .
$$

Therefore,

$$
|H(x, y)| \leq\left.\left.\left.\left.|x-y|^{\frac{1}{r^{\prime}}+\frac{1}{s^{\prime}}}\left|\int_{y}^{x}\right| f^{\prime}(u)\right|^{r} d u\right|^{\frac{1}{r}}\left|\int_{y}^{x}\right| g^{\prime}(w)\right|^{s} d u\right|^{\frac{1}{s}}
$$


Hence,

$$
\begin{aligned}
& 2\left|g_{\rho, \lambda, a+; w}^{\sigma} p(t) \mathcal{g}_{\rho, \lambda, a+; w}^{\sigma} p f g(t)-g_{\rho, \lambda, a+; w}^{\sigma} p g(t) \mathcal{g}_{\rho, \lambda, a+; w}^{\sigma} p f(t)\right| \\
& \leq \int_{a}^{t} \int_{a}^{t}(t-x)^{\lambda-1}(t-y)^{\lambda-1} \mathcal{F}_{\rho, \lambda}^{\sigma}\left[w(t-x)^{\rho}\right] \mathcal{F}_{\rho, \lambda}^{\sigma}\left[w(t-y)^{\rho}\right] p(x) p(y) \\
& \quad \times\left.\left.\left.\left.|x-y|^{\frac{1}{r^{\prime}}+\frac{1}{s^{\prime}}}\left|\int_{y}^{x}\right| f^{\prime}(u)\right|^{r} d u\right|^{\frac{1}{r}}\left|\int_{y}^{x}\right| g^{\prime}(w)\right|^{s} d w\right|^{\frac{1}{s}} d x d y .
\end{aligned}
$$

Again, by using Hölder's inequality, we obtain

$$
\begin{aligned}
& 2\left|\mathcal{g}_{\rho, \lambda, a+; w}^{\sigma} p(t) \mathcal{g}_{\rho, \lambda, a+; w}^{\sigma} p f g(t)-\mathcal{g}_{\rho, \lambda, a+; w}^{\sigma} p g(t) \mathcal{g}_{\rho, \lambda, a+; w}^{\sigma} p f(t)\right| \\
& \leq\left[\int_{a}^{t} \int_{a}^{t}(t-x)^{\lambda-1}(t-y)^{\lambda-1} \mathcal{F}_{\rho, \lambda}^{\sigma}\left[w(t-x)^{\rho}\right] \mathcal{F}_{\rho, \lambda}^{\sigma}\left[w(t-y)^{\rho}\right]\right. \\
& \left.\quad \times\left.\left.|x-y|^{\frac{1}{r^{\prime}}+\frac{1}{s^{\prime}}}\left|\int_{y}^{x}\right| f^{\prime}(u)\right|^{r} d u\right|^{\frac{\gamma}{r}} p(x) p(y) d x d y\right]^{\frac{1}{\gamma}} \\
& \times\left[\int_{a}^{t} \int_{a}^{t}(t-x)^{\lambda-1}(t-y)^{\lambda-1} \mathcal{F}_{\rho, \lambda}^{\sigma}\left[w(t-x)^{\rho}\right] \mathcal{F}_{\rho, \lambda}^{\sigma}\left[w(t-y)^{\rho}\right]\right. \\
& \left.\quad \times\left.\left.|x-y|^{\frac{1}{r^{\prime}}+\frac{1}{s^{\prime}}}\left|\int_{y}^{x}\right| g^{\prime}(w)\right|^{s} d w\right|^{\frac{\gamma}{s}} p(x) p(y) d x d y\right]^{\frac{1}{\gamma}} .
\end{aligned}
$$

Since, we know that

then (2.5) can be written as follows:

$$
\left.\left|\int_{x}^{y}\right| f^{\prime}(u)\right|^{r} d u|\leq|\left|f^{\prime}\right|_{r}^{r},\left.\left.\quad\left|\int_{x}^{y}\right| g^{\prime}(w)\right|^{s} d w\left|\leq \| g^{\prime}\right|\right|_{s} ^{s},
$$

$$
\begin{aligned}
& 2\left|g_{\rho, \lambda, a+; w}^{\sigma} p(t) g_{\rho, \lambda, a+; w}^{\sigma} p f g(t)-g_{\rho, \lambda, a+; w}^{\sigma} p g(t) g_{\rho, \lambda, a+; w}^{\sigma} p f(t)\right| \\
& \leq\left[\left\|f^{\prime}\right\|_{r}^{\gamma} \int_{a}^{t} \int_{a}^{t}(t-x)^{\lambda-1}(t-y)^{\lambda-1} \mathcal{F}_{\rho, \lambda}^{\sigma}\left[w(t-x)^{\rho}\right] \mathcal{F}_{\rho, \lambda}^{\sigma}\left[w(t-y)^{\rho}\right]\right. \\
& \left.\quad \times|x-y|^{\frac{1}{r^{+}}+\frac{1}{s^{\prime}}} p(x) p(y) d x d y\right]^{\frac{1}{\gamma}} \\
& \quad \times\left[\| g^{\prime}||_{s}^{\gamma} \int_{a}^{t} \int_{a}^{t}(t-x)^{\lambda-1}(t-y)^{\lambda-1} \mathcal{F}_{\rho, \lambda}^{\sigma}\left[w(t-x)^{\rho}\right] \mathcal{F}_{\rho, \lambda}^{\sigma}\left[w(t-y)^{\rho}\right]\right. \\
& \left.\quad \times|x-y|^{\frac{1}{r^{+}}+\frac{1}{s^{\prime}}} p(x) p(y) d x d y\right]^{\frac{1}{\gamma}} .
\end{aligned}
$$


Therefore,

$$
\begin{aligned}
& 2\left|g_{\rho, \lambda, a+; w}^{\sigma} p(t) g_{\rho, \lambda, a+; w}^{\sigma} p f g(t)-g_{\rho, \lambda, a+; w}^{\sigma} p g(t) g_{\rho, \lambda, a+; w}^{\sigma} p f(t)\right| \\
& \leq|| f^{\prime}\left|{ }_{r}\right| \mid g^{\prime} \|_{s} \int_{a}^{t} \int_{a}^{t}(t-x)^{\lambda-1}(t-y)^{\lambda-1} \mathcal{F}_{\rho, \lambda}^{\sigma}\left[w(t-x)^{\rho}\right] \mathcal{F}_{\rho, \lambda}^{\sigma}\left[w(t-y)^{\rho}\right] \\
& \quad \times|x-y|^{\frac{1}{r^{\prime}}+\frac{1}{s^{\prime}}} p(x) p(y) d x d y .
\end{aligned}
$$

This completes the proof.

Remark 1. If we choose $\lambda=\alpha, \sigma(0)=1$ and $w=0$ in Theorem 5, then the inequality (2.4) reduces to the inequality (1.1).

We prove also the following result.

Theorem 6. Let $f$ and $g$ be two differentiable functions on $[0, \infty)$ and $p, q$ be positive and integrable function on $[0, \infty)$. If $f^{\prime} \in L_{r}[a, b], g^{\prime} \in L_{s}[a, b], r, s, \gamma>1$ with $\frac{1}{r}+\frac{1}{r^{\prime}}=1, \frac{1}{s}+\frac{1}{s^{\prime}}=1$ and $\frac{1}{\gamma}+\frac{1}{\gamma^{\prime}}=1$, then for all $t>0, \alpha, \rho, w \geq 0$, we have;

$$
\begin{aligned}
& \mid g_{\rho, \lambda, a+; w}^{\sigma} q(t) \mathcal{I}_{\rho, \lambda, a+; w}^{\sigma} p f g(t)+g_{\rho, \lambda, a+; w}^{\sigma} p(t) g_{\rho, \lambda, a+; w}^{\sigma} q f g(t) \\
& \quad-g_{\rho, \lambda, a+; w}^{\sigma} p f(t) g_{\rho, \lambda, a+; w}^{\sigma} q g(t)-g_{\rho, \lambda, a+; w}^{\sigma} q f(t) g_{\rho, \lambda, a+; w}^{\sigma} p g(t) \mid \\
& \leq|| f^{\prime}\left\|_{r}\right\| g^{\prime} \|_{s} \int_{a}^{t} \int_{a}^{t}(t-x)^{\lambda-1}(t-y)^{\lambda-1} \mathcal{F}_{\rho, \lambda}^{\sigma}\left[w(t-x)^{\rho}\right] \mathcal{F}_{\rho, \lambda}^{\sigma}\left[w(t-y)^{\rho}\right] \\
& \quad \times|x-y|^{\frac{1}{r^{\prime}}+\frac{1}{s^{\prime}}} p(x) q(y) d x d y .
\end{aligned}
$$

Proof. We use the same arguments as in the proof of Theorem 5. The proof of this theorem is thus omitted.

Remark 2. If we take $\lambda=\alpha, \sigma(0)=1$ and $w=0$ in Theorem 6 , then the inequality (2.6) reduces to the inequality (1.2).

For the conformable integral approach, we recall the following definition.

Definition 3. [7, Definition $2.1 \&$ Definition 2.2] The left and right-fractional conformable integrals of order $\beta \in \mathbb{C}, \operatorname{Re}(\beta)>0$, are defined by

$$
\begin{aligned}
& { }_{a}^{\beta} I^{\alpha} f(x)=\frac{1}{\Gamma(\beta)} \int_{a}^{x}\left(\frac{(x-a)^{\alpha}-(t-a)^{\alpha}}{\alpha}\right)^{\beta-1} \frac{f(t) d t}{(t-a)^{1-\alpha}}, \\
& { }^{\beta} I_{b}^{\alpha} f(x)=\frac{1}{\Gamma(\beta)} \int_{x}^{b}\left(\frac{(b-x)^{\alpha}-(b-t)^{\alpha}}{\alpha}\right)^{\beta-1} \frac{f(t) d t}{(b-t)^{1-\alpha}} .
\end{aligned}
$$

Notice that, if $(Q f)(t)=f(a+b-t)$, then we have $\left({ }_{a}^{\beta} I^{\alpha} Q f\right)(x)=Q\left({ }^{\beta} I_{b}^{\alpha} f\right)(x)$. Based on this definition, we present to the reader the following two results. 
Theorem 7. Let $f$ and $g$ be two differentiable functions on $[0, \infty)$ and $p$ be positive and integrable function on $[0, \infty)$. If $f^{\prime} \in L_{r}[a, b], g^{\prime} \in L_{s}[a, b], r, s, \gamma>1$ with $\frac{1}{r}+\frac{1}{r^{\prime}}=1, \frac{1}{s}+\frac{1}{s^{\prime}}=1$ and $\frac{1}{\gamma}+\frac{1}{\gamma^{\prime}}=1$, then for all $t>0, \beta \in \mathbb{C}$ and $\operatorname{Re}(\beta)>0$ we have;

$$
\begin{aligned}
& 2\left|{ }_{a}^{\beta} I^{\alpha} p(t){ }_{a}^{\beta} I^{\alpha} p f g(t)-{ }_{a}^{\beta} I^{\alpha} p g(t){ }_{a}^{\beta} I^{\alpha} p f(t)\right| \\
& \leq\left[\frac{\left\|f^{\prime}\right\|_{r}^{\gamma}}{\Gamma(\beta)} \int_{a}^{t} \int_{a}^{t}\left(\frac{(t-a)^{\alpha}-(x-a)^{\alpha}}{\alpha}\right)^{\beta-1}\left(\frac{(t-a)^{\alpha}-(y-a)^{\alpha}}{\alpha}\right)^{\beta-1}\right. \\
& \left.\times \frac{1}{(x-a)^{1-\alpha}} \frac{1}{(y-a)^{1-\alpha}}|x-y|^{\frac{1}{r^{\prime}}+\frac{1}{s^{\prime}}} p(x) p(y) d x d y\right]^{\frac{1}{\gamma}} \\
& \times\left[\frac{\left\|g^{\prime}\right\|_{s}^{\gamma}}{\Gamma(\beta)} \int_{a}^{t} \int_{a}^{t}\left(\frac{(t-a)^{\alpha}-(x-a)^{\alpha}}{\alpha}\right)^{\beta-1}\left(\frac{(t-a)^{\alpha}-(y-a)^{\alpha}}{\alpha}\right)^{\beta-1}\right. \\
& \left.\times \frac{1}{(x-a)^{1-\alpha}} \frac{1}{(y-a)^{1-\alpha}}|x-y|^{\frac{1}{r^{\prime}}+\frac{1}{s^{\prime}}} p(x) p(y) d x d y\right]^{\frac{1}{\gamma}} \\
& \leq \frac{\left\|f^{\prime}\right\|_{r}\left\|g^{\prime}\right\|_{s}}{\Gamma^{2}(\beta)} \int_{a}^{t} \int_{a}^{t}\left(\frac{(t-a)^{\alpha}-(x-a)^{\alpha}}{\alpha}\right)^{\beta-1}\left(\frac{(t-a)^{\alpha}-(y-a)^{\alpha}}{\alpha}\right)^{\beta-1} \\
& \times \frac{1}{(x-a)^{\alpha}} \frac{1}{(y-a)^{\alpha}}|x-y|^{\frac{1}{t^{\prime}}+\frac{1}{s^{\prime}}} p(x) p(y) d x d y .
\end{aligned}
$$

Proof. Let us reconsider the function

$$
H(x, y):=(f(x)-f(y)(g(x)-g(y))) ; \quad x, y \in(0, t), t>0 .
$$

Multiplying (2.8) by $\frac{1}{\Gamma(\beta)}\left(\frac{(t-a)^{\alpha}-(x-a)^{\alpha}}{\alpha}\right)^{\beta-1} \frac{1}{(x-a)^{1-\alpha}} p(x)$ and integrating the resulting identity with respect to $x$ from $a$ to $t$, we can write

$$
\begin{aligned}
\frac{1}{\Gamma(\beta)} \int_{a}^{t}\left(\frac{(t-a)^{\alpha}-(x-a)^{\alpha}}{\alpha}\right)^{\beta-1} \times \frac{1}{(x-a)^{1-\alpha}} p(x) H(x, y) d x \\
\quad={ }_{a}^{\beta} I^{\alpha} p f g(t)-g(y){ }_{a}^{\beta} I^{\alpha} p f(t)-f(y){ }_{a}^{\beta} I^{\alpha} p g(t)+f(y) g(y){ }_{a}^{\beta} I^{\alpha} p(t) .
\end{aligned}
$$

In the same manner, we get:

$$
\begin{aligned}
\frac{1}{\Gamma^{2}(\beta)} \int_{a}^{t} \int_{a}^{t}\left(\frac{(t-a)^{\alpha}-(x-a)^{\alpha}}{\alpha}\right)^{\beta-1}\left(\frac{(t-a)^{\alpha}-(y-a)^{\alpha}}{\alpha}\right)^{\beta-1} \\
\times \frac{1}{(x-a)^{1-\alpha}} \frac{1}{(y-a)^{1-\alpha}} p(x) p(y) H(x, y) d x d y \\
=2\left[{ }_{a}^{\beta} I^{\alpha} p(t){ }_{a}^{\beta} I^{\alpha} p f g(t)-{ }_{a}^{\beta} I^{\alpha} p g(t){ }_{a}^{\beta} I^{\alpha} p f(t)\right] .
\end{aligned}
$$


To achieve the proof, we use the same arguments as in the proof of Theorem 5.

Remark 3. If we choose $a=0$ and $\alpha=1$ in Theorem 7, then the inequality (2.7) reduces to the inequality (1.1).

Theorem 8. Let $f$ and $g$ be two differentiable functions on $[0, \infty)$ and $p, q$ be positive and integrable function on $[0, \infty)$. If $f^{\prime} \in L_{r}[a, b], g^{\prime} \in L_{s}[a, b], r, s, \gamma>1$ with $\frac{1}{r}+\frac{1}{r^{\prime}}=1, \frac{1}{s}+\frac{1}{s^{\prime}}=1$ and $\frac{1}{\gamma}+\frac{1}{\gamma^{\prime}}=1$, then for all $t>0, \beta \in \mathbb{C}$ and $\operatorname{Re}(\beta)>0$, we have

$$
\begin{aligned}
& \mid{ }_{a}^{\beta} I^{\alpha} q(t){ }_{a}^{\beta} I^{\alpha} p f g(t)+{ }_{a}^{\beta} I^{\alpha} p(t){ }_{a}^{\beta} I^{\alpha} q f g(t) \\
& -{ }_{a}^{\beta} I^{\alpha} p f(t){ }_{a}^{\beta} I^{\alpha} q g(t)-{ }_{a}^{\beta} I^{\alpha} q f(t){ }_{a}^{\beta} I^{\alpha} p g(t) \mid \\
& \leq \frac{\left\|f^{\prime}\right\|{ }_{r}\left\|g^{\prime} \mid\right\|_{s}}{\Gamma^{2}(\beta)} \int_{a}^{t} \int_{a}^{t}\left(\frac{(t-a)^{\alpha}-(x-a)^{\alpha}}{\alpha}\right)^{\beta-1}\left(\frac{(t-a)^{\alpha}-(y-a)^{\alpha}}{\alpha}\right)^{\beta-1} \\
& \quad \times \frac{1}{(x-a)^{\alpha}} \frac{1}{(y-a)^{\alpha}}|x-y|^{\frac{1}{r^{+}}+\frac{1}{s^{\prime}}} p(x) q(y) d x d y .
\end{aligned}
$$

Proof. The proof is evident and hence it is omitted.

Remark 4. If we choose $a=0$ and $\alpha=1$ in Theorem 8 , then the inequality (2.9) reduces to the inequality (1.2).

Let us now recall the Saigo integral definition $[8,11,12]$ :

Definition 4. Let $\alpha>0, \beta, \eta \in \mathbb{R}$, then the Saigo fractional integral $\mathrm{J}_{0, t}^{\alpha, \beta, \eta}$ of order $\alpha$ for real-valued continuous function $f(t)$ is defined by

$$
\mathrm{I}_{0, t}^{\alpha, \beta, \eta}\{f(t)\}=\frac{t^{-\alpha-\beta}}{\Gamma(\alpha)} \int_{0}^{t}(t-x)^{\alpha-1}{ }_{2} \mathrm{~F}_{1}\left(\alpha+\beta,-\eta ; \alpha ; 1-\frac{x}{t}\right) f(x) d x,
$$

here, the function ${ }_{2} \mathrm{~F}_{1}(-)$ in the right-hand side of (2.10) is the Gaussian hypergeometric function defined by

$$
{ }_{2} \mathrm{~F}_{1}(a, b ; c ; t)=\sum_{n=0}^{\infty} \frac{(a)_{n}(b)_{n}}{(c)_{n}} \frac{t^{n}}{n !},
$$

and $(a)_{n}$ is the Pochhammer symbol

$$
(a)_{0}=1, \quad(a)_{n}=a(a+1) \ldots(a+n-1) .
$$

Using this definition and with some arguments used in Theorem 5, we can establish the following two results.

Theorem 9. Let $f$ and $g$ be two differentiable functions on $[0, \infty)$ and $p$ be positive and integrable function on $[0, \infty)$. If $f^{\prime} \in L_{r}[a, b], g^{\prime} \in L_{s}[a, b], r, s, \gamma>1$ with 
$\frac{1}{r}+\frac{1}{r^{\prime}}=1, \frac{1}{s}+\frac{1}{s^{\prime}}=1$ and $\frac{1}{\gamma}+\frac{1}{\gamma^{\prime}}=1$, then for all $t>0, \alpha>0$ and $\beta, \eta \in \mathbb{R}$ with $\alpha+\beta \geq 0$ and $\eta \leq 0$ we have

$$
\begin{aligned}
& 2\left|\mathrm{I}_{0, t}^{\alpha, \beta, \eta} p(t) \mathrm{I}_{0, t}^{\alpha, \beta, \eta} p f g(t)-\mathrm{I}_{0, t}^{\alpha, \beta, \eta} p g(t) \mathrm{I}_{0, t}^{\alpha, \beta, \eta} p f(t)\right| \\
& \leq\left[\left\|f^{\prime}\right\|_{r}^{\gamma} \frac{t^{-\alpha-\beta}}{\Gamma(\beta)} \int_{0}^{t} \int_{0}^{t}(t-x)^{\alpha-1}(t-y)^{\alpha-1}{ }_{2} \mathrm{~F}_{1}\left(\alpha+\beta,-\eta ; \alpha ; 1-\frac{x}{t}\right)\right. \\
& \left.\times|x-y|^{\frac{1}{r^{\prime}}+\frac{1}{s^{\prime}}} p(x) p(y) d x d y\right]^{\frac{1}{\gamma}} \\
& \times\left[\left\|g^{\prime}\right\|_{s}^{\gamma} \frac{t^{-\alpha-\beta}}{\Gamma(\beta)} \int_{0}^{t} \int_{0}^{t}(t-x)^{\alpha-1}(t-y)^{\alpha-1}{ }_{2} \mathrm{~F}_{1}\left(\alpha+\beta,-\eta ; \alpha ; 1-\frac{y}{t}\right)\right. \\
& \left.\times|x-y|^{\frac{1}{r^{\prime}}+\frac{1}{s^{\prime}}} p(x) p(y) d x d y\right]^{\frac{1}{\gamma^{\prime}}} \\
& \leq\left\|f^{\prime}\right\|_{r}\left\|g^{\prime}\right\|_{s} \frac{t^{-2 \alpha-2 \beta}}{\Gamma^{2}(\beta)} \int_{0}^{t} \int_{0}^{t}(t-x)^{\alpha-1}(t-y)^{\alpha-1}{ }_{2} \mathrm{~F}_{1}\left(\alpha+\beta,-\eta ; \alpha ; 1-\frac{x}{t}\right) \\
& \times_{2} \mathrm{~F}_{1}\left(\alpha+\beta,-\eta ; \alpha ; 1-\frac{y}{t}\right)|x-y|^{\frac{1}{r^{\prime}}+\frac{1}{s^{\prime}}} p(x) p(y) d x d y .
\end{aligned}
$$

Proof. Let us define

$$
H(x, y):=(f(x)-f(y)(g(x)-g(y))) ; \quad x, y \in(0, t), t>0 .
$$

Multiplying (2.11) by $\frac{t^{-\alpha-\beta}}{\Gamma(\beta)}(t-x)_{2}^{\alpha-1} \mathrm{~F}_{1}\left(\alpha+\beta,-\eta ; \alpha ; 1-\frac{x}{t}\right) p(x)$ and integrating the resulting identity with respect to $x$ from 0 to $t$, we can write

$$
\begin{aligned}
& \frac{t^{-\alpha-\beta}}{\Gamma(\beta)} \int_{0}^{t}(t-x)_{2}^{\alpha-1} \mathrm{~F}_{1}\left(\alpha+\beta,-\eta ; \alpha ; 1-\frac{x}{t}\right) p(x) H(x, y) d x \\
& =\mathrm{I}_{0, t}^{\alpha, \beta, \eta} p f g(t)-g(y) \mathrm{I}_{0, t}^{\alpha, \beta, \eta} p f(t)-f(y) \mathrm{I}_{0, t}^{\alpha, \beta, \eta} p g(t)+f(y) g(y) \mathrm{I}_{0, t}^{\alpha, \beta, \eta} p(t) .
\end{aligned}
$$

Again, multiplying (2.12) by $\frac{t^{-\alpha-\beta}}{\Gamma(\beta)}(t-y)_{2}^{\alpha-1} \mathrm{~F}_{1}\left(\alpha+\beta,-\eta ; \alpha ; 1-\frac{y}{t}\right) p(y)$ and integrating the resulting identity with respect to $y$ from 0 to $t$, we can write

$$
\begin{gathered}
\frac{t^{-2 \alpha-2 \beta}}{\Gamma^{2}(\beta)} \int_{0}^{t} \int_{0}^{t}(t-x)^{\alpha-1}(t-y)_{2}^{\alpha-1} \mathrm{~F}_{1}\left(\alpha+\beta,-\eta ; \alpha ; 1-\frac{x}{t}\right) \\
\quad{ }_{2} \mathrm{~F}_{1}\left(\alpha+\beta,-\eta ; \alpha ; 1-\frac{y}{t}\right) p(x) p(y) H(x, y) d x d y \\
\quad=2\left[\mathrm{I}_{0, t}^{\alpha, \beta, \eta} p(t) \mathrm{I}_{0, t}^{\alpha, \beta, \eta} p f g(t)-\mathrm{I}_{0, t}^{\alpha, \beta, \eta} p g(t) \mathrm{I}_{0, t}^{\alpha, \beta, \eta} p f(t)\right] .
\end{gathered}
$$

Using the same arguments as in the proof of Theorem 5, we obtain the desired result. 
We end this paper by presenting to the reader the following result.

Theorem 10. Let $f$ and $g$ be two differentiable functions on $[0, \infty)$ and $p, q$ be positive and integrable function on $[0, \infty)$. If $f^{\prime} \in L_{r}[a, b], g^{\prime} \in L_{s}[a, b], r, s, \gamma>1$ with $\frac{1}{r}+\frac{1}{r^{\prime}}=1, \frac{1}{s}+\frac{1}{s^{\prime}}=1$ and $\frac{1}{\gamma}+\frac{1}{\gamma^{\prime}}=1$, then for all $t>0, \alpha>0$ and $\beta, \eta \in \mathbb{R}$ with $\alpha+\beta \geq 0$ and $\eta \leq 0$ we have

$$
\begin{aligned}
& \mid \mathrm{I}_{0, t}^{\alpha, \beta, \eta} q(t) \mathrm{I}_{0, t}^{\alpha, \beta, \eta} p f g(t)+\mathrm{I}_{0, t}^{\alpha, \beta, \eta} \mathrm{I}_{0, t}^{\alpha, \beta, \eta} q f g(t) \\
& -\mathrm{I}_{0, t}^{\alpha, \beta, \eta} p f(t) \mathrm{I}_{0, t}^{\alpha, \beta, \eta} q g(t)-\mathrm{I}_{0, t}^{\alpha, \beta, \eta} q f(t) \mathrm{I}_{0, t}^{\alpha, \beta, \eta} p g(t) \mid \\
& \leq\left\|f^{\prime}\right\|_{r} \| g^{\prime}||_{s} \frac{t^{-2 \alpha-2 \beta}}{\Gamma^{2}(\beta)} \int_{0}^{t} \int_{0}^{t}(t-x)^{\alpha-1}(t-y)^{\alpha-1}{ }_{2} \mathrm{~F}_{1}\left(\alpha+\beta,-\eta ; \alpha ; 1-\frac{x}{t}\right) \\
& \quad \times_{2} \mathrm{~F}_{1}\left(\alpha+\beta,-\eta ; \alpha ; 1-\frac{y}{t}\right)|x-y|^{\frac{1}{r^{+}}+\frac{1}{s^{\prime}}} p(x) q(y) d x d y .
\end{aligned}
$$

Proof. Multiplying (2.12) by $\frac{t^{-\alpha-\beta}}{\Gamma(\beta)}(t-y)^{\alpha-1}{ }_{2} \mathrm{~F}_{1}\left(\alpha+\beta,-\eta ; \alpha ; 1-\frac{y}{t}\right) q(y)$ and integrating the resulting identity with respect to $y$ from $a$ to $t$, we can write

$$
\begin{aligned}
& \mid \mathrm{I}_{0, t}^{\alpha, \beta, \eta} q(t) \mathrm{I}_{0, t}^{\alpha, \beta, \eta} p f g(t)+\mathrm{I}_{0, t}^{\alpha, \beta, \eta} p(t) \mathrm{I}_{0, t}^{\alpha, \beta, \eta} q f g(t) \\
& -\mathrm{I}_{0, t}^{\alpha, \beta, \eta} p f(t) \mathrm{I}_{0, t}^{\alpha, \beta, \eta} q g(t)-\mathrm{I}_{0, t}^{\alpha, \beta, \eta} q f(t) \mathrm{I}_{0, t}^{\alpha, \beta, \eta} p g(t) \mid \\
& \leq \frac{t^{-2 \alpha-2 \beta}}{\Gamma^{2}(\beta)} \int_{0}^{t} \int_{0}^{t}(t-x)^{\alpha-1}(t-y)^{\alpha-1}{ }_{2} \mathrm{~F}_{1}\left(\alpha+\beta,-\eta ; \alpha ; 1-\frac{x}{t}\right) \\
& \times|x-y|^{\frac{1}{r}}+\left.\left.\left.\left.\frac{1}{s^{\prime}}\left|\int_{y}^{x}\right| f^{\prime}(u)\right|^{r} d u\right|^{\frac{1}{r}}\left|\int_{y}^{x}\right| g^{\prime}(w)\right|^{s} d w\right|^{\frac{1}{s}} p(x) q(y) d x d y .
\end{aligned}
$$

Some easy calculations allow us to achieve the proof of this theorem.

\section{CONCLUSiON}

In this paper, we presented several fractional integral inequalities related to the weighted and the extended Chebyshev functional using generalized fractional integral operators, new conformable fractional integral operators and Saigo fractional integral operator, respectively. The results exhibited in Section 2 generalized the work earlier done by Dahmani [4] for Riemann-Liouville fractional integral operator. 


\section{REFERENCES}

[1] R. P. Agarwal, M.-J. Luo, and R. K. Raina, "On Ostrowski type inequalities," Fasciculi Mathematici, vol. 56, no. 1, pp. 5-27, jun 2016, doi: 10.1515/fascmath-2016-0001.

[2] P. L. Chebyshev, "Sur les expressions approximatives des integrales definies par les autres prisesentre les mêmes limites," Proc. Math. Soc. Charkov, vol. 2, pp. 93-98, 1882.

[3] Z. Dahmani, "New inequalities in fractional integrals," International Journal of Nonlinear Science, vol. 9, no. 4, pp. 493-497, 2010.

[4] Z. Dahmani, A. Khameli, and K. Freha, "Some RL-integral inequalities for the weighted and extended Chebyshev functionals," Konuralp Journal of Mathematics, vol. 5, no. 1, pp. 43-48, 2017.

[5] Z. Dahmani, O. Mechouar, and S. Brahami, "Certain inequalities related to the Chebyshev's functional involving a Riemann-Liouville operator,” Bull. Math. Anal. Appl., vol. 3, no. 4, pp. 38-44, 2011.

[6] N. Elezović, L. bo Marangunić, and J. Pečarić, "Some improvements of Grüss type inequality," Journal of Mathematical Inequalities, no. 3, pp. 425-436, 2007, doi: 10.7153/jmi-01-36.

[7] F. Jarad, E. Uğurlu, T. Abdeljawad, and D. Baleanu, "On a new class of fractional operators," Advances in Difference Equations, vol. 2017, no. 1, aug 2017, doi: 10.1186/s13662-017-1306-z.

[8] V. Kiryakova, Generalized Fractional Calculus and Applications, vol. 301 of Pitman Research Notes in Mathematics Series. Longman Scientific \& Technical, Harlow, UK, 1994.

[9] D. S. Mitrinovic, Analytic inequalities. S Springer Verlag, Berlin, 1970.

[10] R. K. Raina, "On generalized Wright's hypergeometric functions and fractional calculus operators," East Asian Math. J., vol. 21, no. 2, pp. 191-203, 2005.

[11] R. K. Raina, "Solution of Abel-type integral equation involving the Appell hypergeometric function," Integral Transforms and Special Functions, vol. 21, no. 7, pp. 515-522, jul 2010, doi: 10.1080/10652460903403547.

[12] M. Saigo, "A remark on integral operators involving the Gauss hypergeometric functions," Math. Rep. Kyushu Univ., vol. 11, pp. 135-143, 1978.

[13] E. Set, A. Akdemir, and B. Çelik, "On generalization of Fejér type inequalities via fractional integral operators," Filomat, vol. 32, no. 16, pp. 5537-5547, 2018, doi: 10.2298/FIL1816537S.

[14] E. Set and B. Çelik, "Generalized fractional Hermite-Hadamard type inequalities for m-convex and $(\alpha, m)$-convex functions," Communications Faculty Of Science University of Ankara Series AlMathematics and Statistics, vol. 67, no. 1, pp. 333-344, 2018.

[15] E. Set and A. Gözpınar, "Some new inequalities involving generalized fractional integral operators for several class of functions," AIP Conf. Proc., vol. 1833, no. 1, 2017, doi: 10.1063/1.4981686.

[16] E. Set, M. A. Noor, M. U. Awan, and A. Gözpinar, "Generalized Hermite-Hadamard type inequalities involving fractional integral operators," Journal of Inequalities and Applications, vol. 2017, no. 1, jul 2017, doi: 10.1186/s13660-017-1444-6.

[17] E. Set, M. Z. Sarikaya, M. E. Özdemir, and H. Yıldırım, "The Hermite-Hadamard's inequality for some convex functions via fractional integrals and related results," Journal of Applied Mathematics, Statistics and Informatics, vol. 10, no. 2, pp. 69-83, dec 2014, doi: 10.2478/jamsi-2014-0014.

Authors' addresses

Emrullah Aykan Alan

Ordu University, Department of Mathematics, Faculty of Science and Arts, Ordu, Turkey

E-mail address: aykanalan@gmail.com 


\section{Barış Çelik}

Ordu University, Department of Mathematics, Faculty of Science and Arts, Ordu, Turkey

E-mail address: bariscelik15 @hotmail.com

Erhan Set

(Corresponding author) Ordu University, Department of Mathematics, Faculty of Science and Arts, Ordu, Turkey

E-mail address: erhanset@yahoo.com

Zoubir Dahmani

University of Mostaganem, Faculty of SEI, Laboratory of Pure and Applied Mathematics, Mostaganem, Algeria

E-mail address: zzdahmani@yahoo.fr 